\title{
Turing Patterns in a Predator-Prey System with Self-Diffusion
}

\author{
Hongwei Yin, Xiaoyong Xiao, and Xiaoqing Wen \\ School of Science, Nanchang University, Nanchang 330031, China \\ Correspondence should be addressed to Hongwei Yin; hongwei-yin@hotmail.com
}

Received 24 June 2013; Revised 17 September 2013; Accepted 2 October 2013

Academic Editor: Francisco Solís Lozano

Copyright (C) 2013 Hongwei Yin et al. This is an open access article distributed under the Creative Commons Attribution License, which permits unrestricted use, distribution, and reproduction in any medium, provided the original work is properly cited.

\begin{abstract}
For a predator-prey system, cross-diffusion has been confirmed to emerge Turing patterns. However, in the real world, the tendency for prey and predators moving along the direction of lower density of their own species, called self-diffusion, should be considered. For this, we investigate Turing instability for a predator-prey system with nonlinear diffusion terms including the normal diffusion, cross-diffusion, and self-diffusion. A sufficient condition of Turing instability for this system is obtained by analyzing the linear stability of spatial homogeneous equilibrium state of this model. A series of numerical simulations reveal Turing parameter regions of the interaction of diffusion parameters. According to these regions, we further demonstrate dispersion relations and spatial patterns. Our results indicate that self-diffusion plays an important role in the spatial patterns.
\end{abstract}

\section{Introduction}

In ecological systems, the interactions of different species indicate abundant dynamical features. It is informative to use mathematical model to study the interactions of species in these systems. Among these models, predator-prey systems, which were based on the pioneering works of Volterra [1], have been important in ecological problems. However, since we live in a spatial world, the predator-prey systems should include spatial factors. Thus, these systems should be described by using reaction-diffusion equations. As a result, it is an open problem to understand spatiotemporal behaviors of the temporal-spatial predator-prey systems [211]. Thereinto, the formation of spatial patterns of predatorprey systems is a very active research area [12-15], which is based on the pioneering work of Turing [16] in 1952.

In recent years, there are a lot of bodies of literature to study the predator-prey system by taking into account the normal diffusion as well as cross-diffusion [17-20]. Normal diffusion is a natural phenomenon of the movement of the prey or the predators from higher-density regions to lowerdensity ones. Cross-diffusion of the prey expresses a flux of the prey because of the presence of the predators and vice versa. Furthermore, in predator-prey systems, cross-diffusion can induce Turing instability to produce spatial patterns even though spatial homogeneous equilibrium states for the corresponding system in the absence of cross-diffusion are stable $[8,11,21-28]$.

Besides the normal diffusion and the cross-diffusion of the predator and prey in ecological systems, there exists, in fact, another diffusion form-self-diffusion for the pressure of their own species. It can describe the tendency to move along the direction of lower density of the predator's and prey's own species [29]. Unfortunately, most of the studies mainly focused on well-posedness of solutions for predatorprey systems with self-diffusion [30, 31]. Little attention was paid to examine Turing patterns of these systems. Based on the above discussion, in this paper we mainly concentrate on Turing instability of a predator-prey system that includes a normal diffusion, cross-diffusion, and self-diffusion terms. To this end, we find a sufficient condition to generate Turing patterns. By using numerical simulation, for this system we examine parameter regions of forming patterns and show snapshots of spatial patterns.

The paper is organized as follows. In Section 2, we build the predator-prey model with nonlinear diffusion terms including normal diffusion, cross-diffusion, and selfdiffusion terms and the biological meaning of these parameters are interpreted. In Section 3, we find the sufficient condition of Turing instability. By performing a series of 
numerical simulations, we locate the Turing parameter spaces when parts of the parameters are fixed in Section 4. In Section 5, by choosing values of some parameters from the Turing parameter spaces, we illustrate Turing patterns. Finally, some conclusions and discussions are given.

\section{A Predator-Prey Model with Nonlinear Diffusion}

In this paper, we are interested in the spatiotemporal patterns of the following predator-prey system with nonlineardiffusion terms. Mathematical properties for this system have been investigated in $[30,31]$ :

$$
\begin{gathered}
\frac{\partial u_{1}}{\partial t}-\Delta\left[\left(d_{1}+a_{11} u_{1}+a_{12} u_{2}\right) u_{1}\right] \\
=u_{1}\left(a_{1}-b_{1} u_{1}-c_{1} u_{2}\right), \quad \text { in } \Omega \times[0, \infty), \\
\frac{\partial u_{2}}{\partial t}-\Delta\left[\left(d_{2}+a_{21} u_{1}+a_{22} u_{2}\right) u_{2}\right] \\
=u_{2}\left(a_{2}+b_{2} u_{1}-c_{2} u_{2}\right), \quad \text { in } \Omega \times[0, \infty), \\
\frac{\partial u_{1}}{\partial n}=\frac{\partial u_{2}}{\partial n}=0, \quad \text { on } \partial \Omega \times[0, \infty), \\
u_{1}(x, 0)=u_{1}^{0}(x) \geq 0, \\
u_{2}(x, 0)=u_{2}^{0}(x) \geq 0, \quad \text { in } \Omega,
\end{gathered}
$$

where $\Omega$ is a bounded domain in $\mathbb{R}^{2}$ with smooth boundary $\partial \Omega$ and represents the domain that these two species inhabit. The vector $n$ is the outward unit normal vector of $\Omega$. In this model, $u_{1}$ and $u_{2}$ are the prey and the predator densities, respectively; $a_{i}, b_{i}$, and $c_{i}(i=1,2)$ are positive constants. $a_{1}, a_{2}$ are rates of the prey and predator proliferations for food source; $a_{1} / b_{1}$ and $a_{2} / c_{2}$ are environmental carrying capacities for prey and predator, respectively. $c_{1}$ is a consumption rate; $b_{2}$ is a conversion rate. In the diffusion terms, the constant $d_{i}(i=1,2)$, which is usually termed as a normaldiffusion coefficient, represents the natural dispersive force of movement of a species. The positive constants $a_{12}$ and $a_{21}$ are referred to as cross-diffusion coefficients, which describe that the prey tends to avoid higher density of the predators and vice versa by diffusing away. In addition, for the predators and the prey, the positive constants $a_{11}$ and $a_{22}$ are self-diffusion rates due to pressure within their own species.

Next, we want to look for the condition on the parameter values such that a positive homogeneous equilibrium state is linearly stable in the absence of the cross-diffusion and the self-diffusion (i.e., a normal reaction-diffusion system) but unstable in the present of the cross-diffusion and the selfdiffusion. 24].

For simplicity, we set up the following notation as in [22,

Notation 1. Let $0=\mu_{1}<\mu_{2}<\cdots \rightarrow \infty$ be the eigenvalues of $-\Delta$ on $\Omega$ under no-flux boundary condition, and let $E\left(\mu_{i}\right)$ be the space of eigenfunction corresponding to $\mu_{i}$. We define the following space decomposition:

(i) $\mathbf{X}_{i j}:=\left\{\varphi_{i j} \mathbf{c}: \mathbf{c} \in \mathbb{R}^{2}\right\}$, where $\varphi_{i j}$ are orthonormal bases of $E\left(\mu_{i}\right)$ for $j=1, \ldots, \operatorname{dim} E\left(\mu_{i}\right)$;

(ii) $\mathbf{X}:=\left\{\mathbf{u} \in\left[\mathscr{C}^{1}(\bar{\Omega})\right]^{2}: \partial u_{1} / \partial n=\partial u_{2} / \partial n=0\right.$ on $\left.\partial \Omega\right\}$, and thus $\mathbf{X}=\oplus_{i=1}^{\infty} \mathbf{X}_{i}$, where $\mathbf{X}_{i}=\oplus_{j=1}^{\operatorname{dim} E\left(\mu_{i}\right)} \mathbf{X}_{i j}$.

Notation 2. For the sake of simplicity, we denote reaction terms for systems (1) by

$$
\mathbf{G}(\mathbf{u})=\left(\begin{array}{l}
G_{1}(\mathbf{u}) \\
G_{2}(\mathbf{u})
\end{array}\right)=\left(\begin{array}{l}
u_{1} g_{1}(\mathbf{u}):=u_{1}\left(a_{1}-b_{1} u_{1}-c_{1} u_{2}\right) \\
u_{2} g_{2}(\mathbf{u}):=u_{2}\left(a_{2}+b_{2} u_{1}-c_{2} u_{2}\right)
\end{array}\right),
$$

and its Jacobian matrix at the point $\mathbf{u}^{*}$ is

$$
\mathbf{G}_{\mathbf{u}}\left(\mathbf{u}^{*}\right)=\left(\begin{array}{ll}
G_{11} & G_{12} \\
G_{21} & G_{22}
\end{array}\right):=\left(\begin{array}{cc}
-b_{1} u_{1}^{*} & -c_{1} u_{1}^{*} \\
b_{2} u_{2}^{*} & -c_{2} u_{2}^{*}
\end{array}\right) .
$$

The diffusion term is denoted as

$$
\begin{aligned}
\Phi(\mathbf{u}) & =\left(\begin{array}{l}
\Phi_{1}(\mathbf{u}) \\
\Phi_{2}(\mathbf{u})
\end{array}\right) \\
& :=\left(\begin{array}{l}
\left(d_{1}+a_{11} u_{1}+a_{12} u_{2}\right) u_{1} \\
\left(d_{2}+a_{21} u_{1}+a_{22} u_{2}\right) u_{2}
\end{array}\right),
\end{aligned}
$$

and its corresponding Jacobian matrix at the point $\mathbf{u}^{*}$ is

$$
\begin{aligned}
\Phi_{\mathbf{u}}\left(\mathbf{u}^{*}\right) & =\left(\begin{array}{ll}
\Phi_{11} & \Phi_{12} \\
\Phi_{21} & \Phi_{22}
\end{array}\right) \\
& :=\left(\begin{array}{cc}
d_{1}+2 a_{11} u_{1}^{*}+a_{12} u_{2}^{*} & a_{12} u_{1}^{*} \\
a_{21} u_{2}^{*} & d_{2}+a_{21} u_{1}^{*}+2 a_{22} u_{2}^{*}
\end{array}\right) .
\end{aligned}
$$

\section{Linear Stability Analysis of System (1) with a Normal Diffusion}

Let $a_{i j}=0$ for $i, j=1,2$; then the system (1) degenerates into standard reaction-diffusion equations:

$$
\begin{gathered}
\frac{\partial u_{1}}{\partial t}-d_{1} \Delta u_{1}=u_{1}\left(a_{1}-b_{1} u_{1}-c_{1} u_{2}\right), \quad \text { in } \Omega \times[0, \infty), \\
\frac{\partial u_{2}}{\partial t}-d_{2} \Delta u_{2}=u_{2}\left(a_{2}+b_{2} u_{1}-c_{2} u_{2}\right), \quad \text { in } \Omega \times[0, \infty), \\
\frac{\partial u_{1}}{\partial n}=\frac{\partial u_{2}}{\partial n}=0, \quad \text { on } \partial \Omega \times[0, \infty), \\
u_{1}(x, 0)=u_{1}^{0}(x) \geq 0, \\
u_{2}(x, 0)=u_{2}^{0}(x) \geq 0, \quad \text { in } \Omega .
\end{gathered}
$$

Through this paper, we assume the following conditions:

$$
[C 1] \frac{c_{2}}{c_{1}}>\frac{a_{2}}{a_{1}}, \quad[C 2] \quad \frac{b_{2}}{b_{1}}>\frac{c_{2}}{c_{1}}-\frac{2 a_{2}}{a_{1}} .
$$


Then, there exists a unique positive equilibrium state for system (6), denoted by $\mathbf{u}^{*}=\left(u_{1}^{*}, u_{2}^{*}\right)$, where

$$
u_{1}^{*}=\frac{a_{1} c_{2}-a_{2} c_{1}}{b_{1} c_{2}+b_{2} c_{1}}, \quad u_{2}^{*}=\frac{a_{1} b_{2}+a_{2} b_{1}}{b_{1} c_{2}+b_{2} c_{1}} .
$$

Theorem 1. If there are no cross-diffusion and self-diffusion, the positive equilibrium state $\mathbf{u}^{*}$ of the system (6) is locally asymptotically stable when condition $[\mathrm{C} 1]$ holds.

Proof. The linearization of (6) around the steady state $\mathbf{u}^{*}$ can be therefore expressed by

$$
\frac{\partial \mathbf{u}}{\partial t}=\left(D \Delta+\mathbf{G}_{\mathbf{u}}\left(\mathbf{u}^{*}\right)\right) \mathbf{u},
$$

where $D=\operatorname{diag}\left(d_{1}, d_{2}\right)$. Obviously, the operator $D \Delta+\mathbf{G}_{\mathbf{u}}\left(\mathbf{u}^{*}\right)$ is invariant in the subspace $\mathbf{X}_{i}$, and $\lambda_{i}$ is an eigenvalue of this operator on $\mathbf{X}_{i}$, if and only if it is an eigenvalue of $-\mu_{i} D+$ $\mathbf{G}_{\mathbf{u}}\left(\mathbf{u}^{*}\right)$. Direct calculation shows the characteristic equation

$$
\lambda_{i}^{2}-m_{i} \lambda_{i}+n_{i}=0
$$

where $m_{i}=-u_{1}^{*} b_{1}-u_{2}^{*} c_{2}-\left(d_{1}+d_{2}\right) \mu_{i}$ and $n_{i}=\left(u_{1}^{*} b_{1}+\right.$ $\left.d_{1} \mu_{i}\right)\left(u_{2}^{*} c_{2}+d_{2} \mu_{i}\right)+u_{1}^{*} u_{2}^{*} b_{2} c_{1}$. It is easy to be verified that $m_{i}$ is negative and $n_{i}$ positive. Thus, for each $i>1$, the two roots of (10) have negative real parts. Consequently, we complete this proof.

Remark 2. According to the proof of Theorem 1, we can also calculate out the eigenvector $\mathbf{c}$ corresponding to the eigenvalue $\lambda_{i}$ for the operator $-\mu_{i} D+\mathbf{G}_{\mathbf{u}}\left(\mathbf{u}^{*}\right)$, which satisfies

$$
\left(\lambda_{i}+\mu_{i} D-\mathbf{G}_{\mathbf{u}}\left(\mathbf{u}^{*}\right)\right) \mathbf{c}=0 .
$$

Furthermore, we can yield corresponding eigenspace $\left\{\varphi_{i j} \mathbf{c}\right\}$ in $\mathrm{X}_{i}$.

By Theorem 1, under condition [C1] system (6) cannot destabilize $\mathbf{u}^{*}$. Next, the cross-diffusion and self-diffusion are taken into account.

Theorem 3. Assume that conditions [C1] and [C2] hold; then there exists a sufficiently large positive constant $a_{21}^{*}$ such that the homogeneous state $\mathbf{u}^{*}$ of the system (1) is unstable provided that $a_{21}>a_{21}^{*}$.

Proof. We first linearize the system (1) around $\left(u_{1}, u_{2}\right)=$ $\left(u_{1}^{*}, u_{2}^{*}\right)$ and arrive at

$$
\frac{\partial \mathbf{u}}{\partial t}=\left(\Phi_{\mathbf{u}}\left(\mathbf{u}^{*}\right) \Delta+\mathbf{G}_{\mathbf{u}}\left(\mathbf{u}^{*}\right)\right) \mathbf{u} .
$$

By the same method as the proof of Theorem 1, we consider the operator $\Phi_{\mathbf{u}}\left(\mathbf{u}^{*}\right) \Delta+\mathbf{G}_{\mathbf{u}}\left(\mathbf{u}^{*}\right)$ on the subspace $\mathbf{X}_{i}$. The eigenvalue of this operator on $\mathbf{X}_{i}$ is denoted as $\lambda$, and then it is also an eigenvalue of the matrix $-\mu_{i} \Phi_{\mathbf{u}}\left(\mathbf{u}^{*}\right)+\mathbf{G}_{\mathbf{u}}\left(\mathbf{u}^{*}\right)$. Thus, $\lambda$ satisfies the following equation:

$$
\begin{array}{r}
\lambda^{2}-\left[G_{11}+G_{22}-\mu_{i}\left(\Phi_{11}+\Phi_{22}\right)\right] \lambda \\
+\operatorname{det}\left(\mathbf{G}_{\mathbf{u}}\left(\mathbf{u}^{*}\right)-\mu_{i} \Phi_{\mathbf{u}}\right)=0 .
\end{array}
$$

According to Notation 2, we can calculate and get

$$
\begin{aligned}
G_{11}+ & G_{22}-\mu_{i}\left(\Phi_{11}+\Phi_{22}\right) \\
= & -\mu_{i}\left[d_{1}+d_{2}+\left(2 a_{11}+a_{21}\right) u_{1}^{*}+\left(a_{12}+2 a_{12}\right) u_{2}^{*}\right] \\
& -b_{1} u_{1}^{*}-c_{2} u_{2}^{*}<0 \\
& \operatorname{det}\left(\mathbf{G}_{\mathbf{u}}\left(\mathbf{u}^{*}\right)-\mu_{i} \Phi_{\mathbf{u}}\right)=a \mu_{i}^{2}+b \mu_{i}+c
\end{aligned}
$$

where

$$
\begin{aligned}
a= & d_{1} d_{2}+d_{1}\left(a_{21} u_{1}^{*}+2 a_{22} u_{2}^{*}\right) \\
& +d_{2}\left(2 a_{11} u_{1}^{*}+a_{12} u_{2}^{*}\right)+2 a_{11} a_{21}\left(u_{1}^{*}\right)^{2} \\
& +4 a_{11} a_{22} u_{1}^{*} u_{2}^{*}+2 a_{12} a_{22}\left(u_{2}^{*}\right)^{2}>0, \\
b= & c_{2} u_{2}^{*}\left(d_{1}+2 a_{11} u_{1}^{*}+a_{12} u_{2}^{*}\right) \\
& +b_{1} u_{1}^{*}\left(d_{2}+a_{21} u_{1}^{*}+2 a_{22} u_{2}^{*}\right) \\
& +a_{12} b_{2} u_{1}^{*} u_{2}^{*}-a_{21} c_{1} u_{1}^{*} u_{2}^{*}, \\
c= & b_{1} c_{2} u_{1}^{*} u_{2}^{*}+b_{2} c_{1} u_{1}^{*} u_{2}^{*}>0 .
\end{aligned}
$$

Let $\lambda_{1}\left(\mu_{i}\right)$ and $\lambda_{2}\left(\mu_{i}\right)$ be the two roots of (13); then we have

$$
\begin{gathered}
\lambda_{1}\left(\mu_{i}\right)+\lambda_{2}\left(\mu_{i}\right)=G_{11}+G_{22}-\mu_{i}\left(\Phi_{11}+\Phi_{22}\right), \\
\lambda_{1}\left(\mu_{i}\right) \lambda_{2}\left(\mu_{i}\right)=\operatorname{det}\left(\mathbf{G}_{\mathbf{u}}\left(\mathbf{u}^{*}\right)-\mu_{i} \Phi_{\mathbf{u}}\right) .
\end{gathered}
$$

In order to ensure that $\operatorname{Re} \lambda_{1}\left(\mu_{i}\right)<0$ and $\operatorname{Re} \lambda_{2}\left(\mu_{i}\right)>0$, a sufficient condition of Turing instability about homogeneous equilibrium $\mathbf{u}^{*}$ is

$$
\operatorname{det}\left(\mathbf{G}_{\mathbf{u}}\left(\mathbf{u}^{*}\right)-\mu_{i} \Phi_{\mathbf{u}}\right)<0 .
$$

Next, we look for the diffusion conditions such that $\operatorname{det}\left(\mathbf{G}_{\mathbf{u}}\left(\mathbf{u}^{*}\right)-\mu_{i} \Phi_{\mathbf{u}}\right)<0$ holds. Furthermore, we have

$$
\begin{gathered}
\lim _{a_{21} \rightarrow \infty} \frac{a}{a_{21}}=\left(d_{1}+2 a_{11} u_{1}^{*}\right) u_{1}^{*}, \\
\lim _{a_{21} \rightarrow \infty} \frac{b}{a_{21}}=\left(b_{1} u_{1}^{*}-c_{1} u_{2}^{*}\right) u_{1}^{*}, \\
\lim _{a_{21} \rightarrow \infty} \frac{c}{a_{21}}=0 .
\end{gathered}
$$

Taking condition [C2] in consideration, we can obtain that

$$
\begin{gathered}
\lim _{a_{21} \rightarrow \infty} \frac{\operatorname{det}\left(\mathbf{G}_{\mathbf{u}}\left(\mathbf{u}^{*}\right)-\mu_{i} \Phi_{\mathbf{u}}\right)}{a_{21}}=\left(d_{1}+2 a_{11} u_{1}^{*}\right) u_{1}^{*} \mu_{i}^{2} \\
+\left(b_{1} u_{1}^{*}-c_{1} u_{2}^{*}\right) u_{1}^{*} \mu_{i}:=P\left(\mu_{i}\right) .
\end{gathered}
$$

Then, $P\left(\mu_{i}\right)=0$ has two real roots, one being 0 and the other being positive. A continuity argument shows that there exists a positive constant $a_{21}^{*}$ such that when $a_{21}>a_{21}^{*}$, (15) and $\mu_{i}$ axis intersect on two real and positive points, denoted by $\mu_{i 1}$, $\mu_{i 2}\left(\mu_{i 1}<\mu_{i 2}\right)$. Hence, there exists a $\mu_{i} \in\left(\mu_{i 1}, \mu_{i 2}\right)$ such that $\operatorname{det}\left(\mathbf{G}_{\mathbf{u}}\left(\mathbf{u}^{*}\right)-\mu_{i} \Phi_{\mathbf{u}}\right)<0$. 


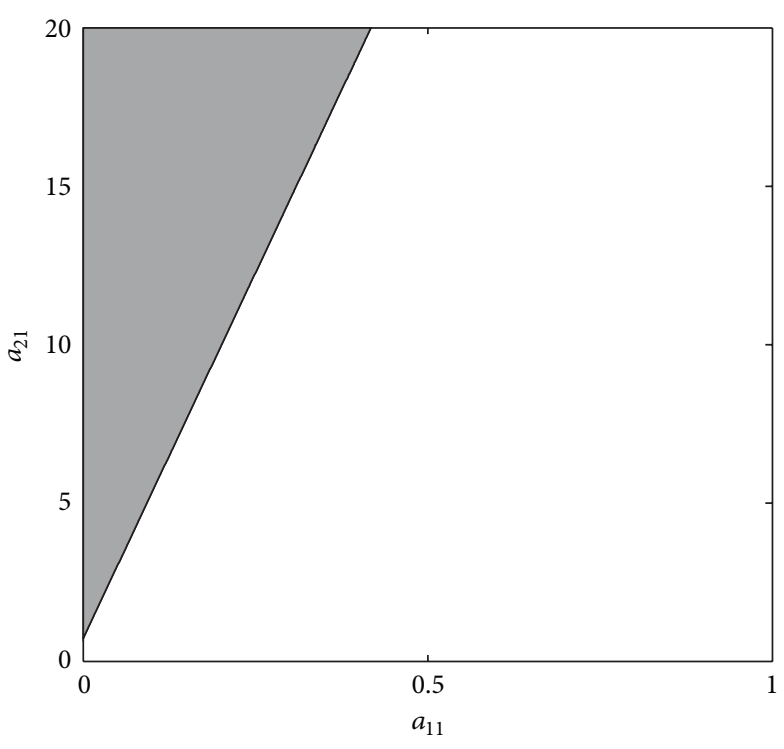

(a)

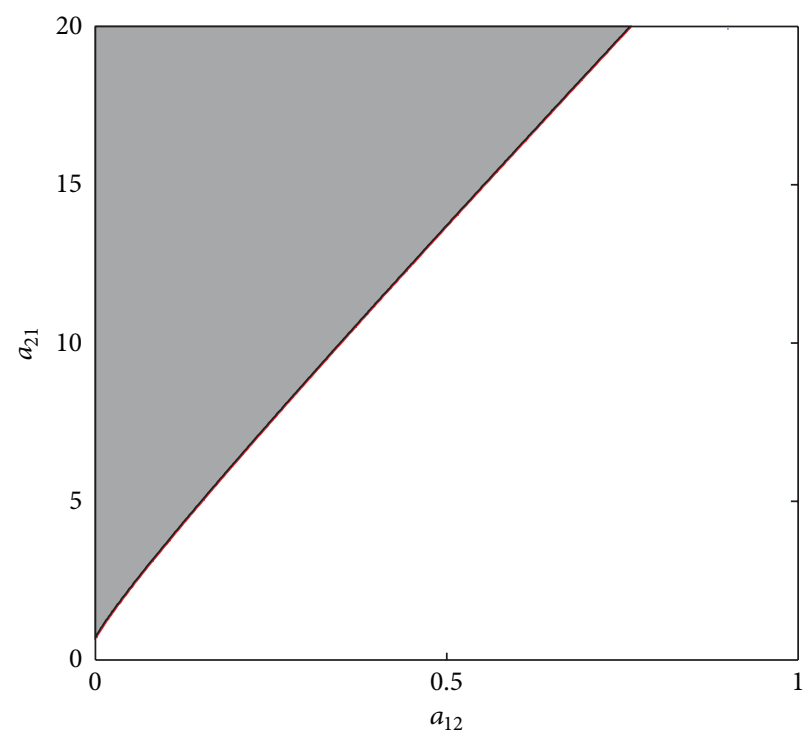

(b)

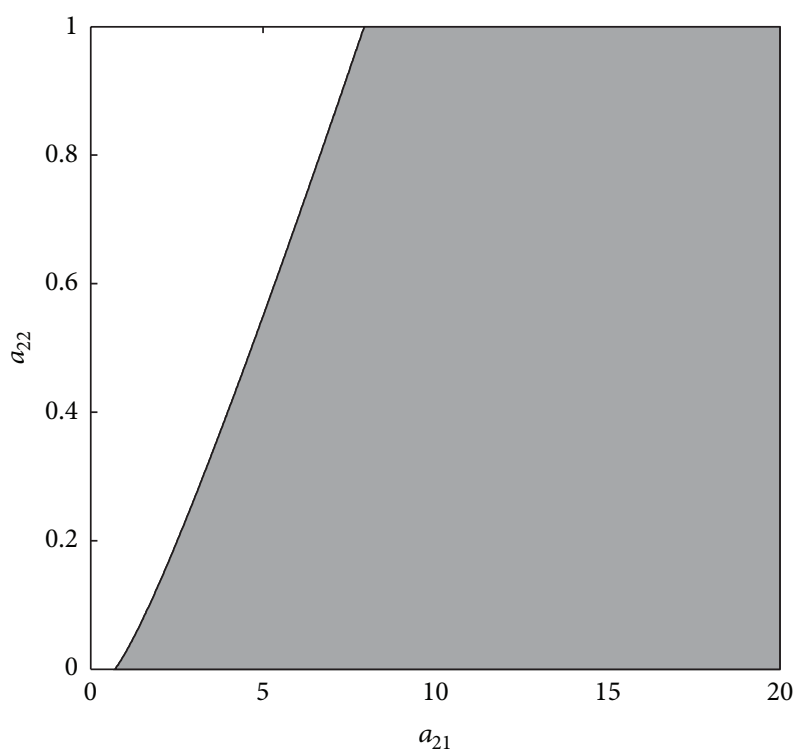

(c)

FIgURE 1: Turing parameter spaces for the system (1). Inside the gray zones, $\mathbf{u}^{*}$ is unstable, while, for parameters outside these zones, $\mathbf{u}^{*}$ remains stable. These graphics are obtained by fixing parameters in (21); besides $a_{12}=a_{22}=0.01(\mathrm{a}), a_{11}=a_{22}=0.01(\mathrm{~b})$, and $a_{11}=a_{12}=0.01$ (c).

Remark 4. Theorem 3 is available for a case of the system (1) equipped with cross-diffusion and self-diffusion; that is, $a_{11} \neq 0, a_{12} \neq 0$ and $a_{22} \neq 0$. When $a_{11}=a_{12}=a_{22}=0$ and $a_{21} \neq 0$, the system (1) possesses a diffusion term the same as in [22].

Corollary 5. If $a_{21}=0$, then the homogeneous steady state $\mathbf{u}^{*}$ of the system (1) is always stable.

\section{Turing Parameter Space}

In this section, we will find some parameter regions of nonlinear diffusion coefficients where the equilibrium state $\mathbf{u}^{*}$ is unstable. For this, according to (15) the sufficient condition of Turing instability is $b<0$ and $b^{2}-4 a c>0$ besides $[C 1]$ and $[C 2]$. In this paper, the parameter values satisfying conditions $[\mathrm{C} 1]$ and $[\mathrm{C} 2]$ are taken as follows:

$$
\begin{array}{ccc}
a_{1}=1, & a_{2}=2, & b_{1}=0.08, \\
b_{2}=0.05, & c_{1}=0.05, & c_{2}=0.15, \\
& d_{1}=d_{2}=0.1 .
\end{array}
$$

Then, for these fixed parameters the homogeneous steady state $\mathbf{u}^{*}$ is given by $\left(u_{1}^{*}, u_{2}^{*}\right)=(3.4483,14.4828)$. In Figure 1, we examine the parameter regions where 


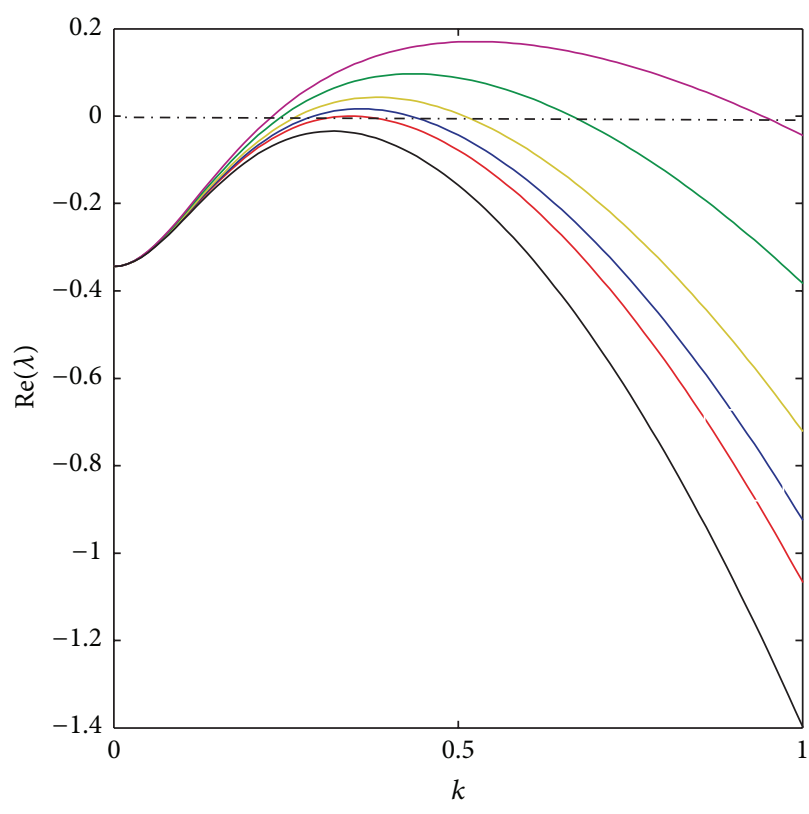

(a)

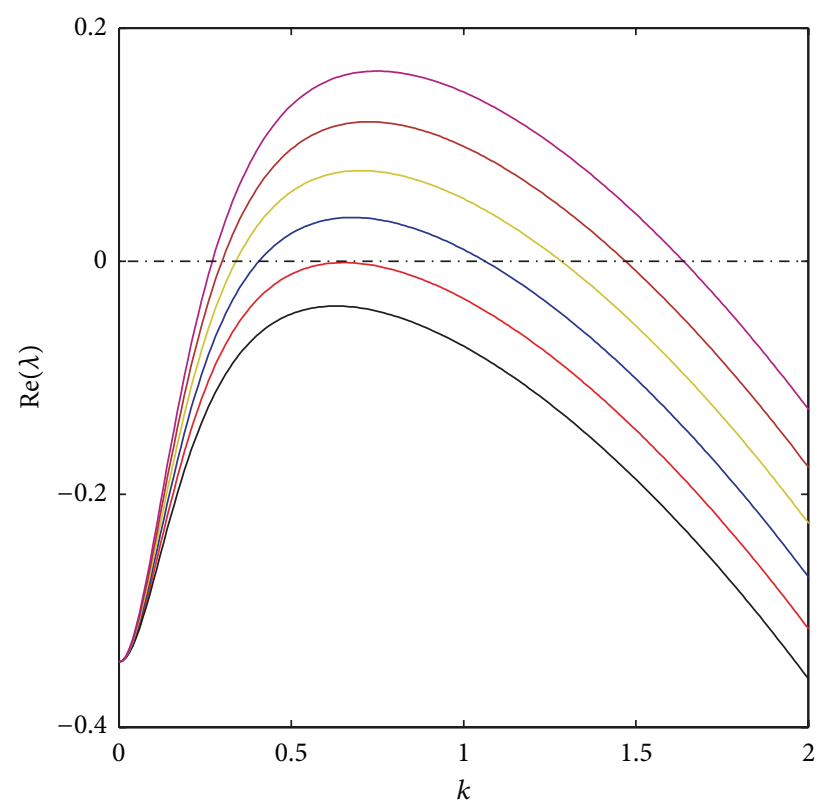

(b)

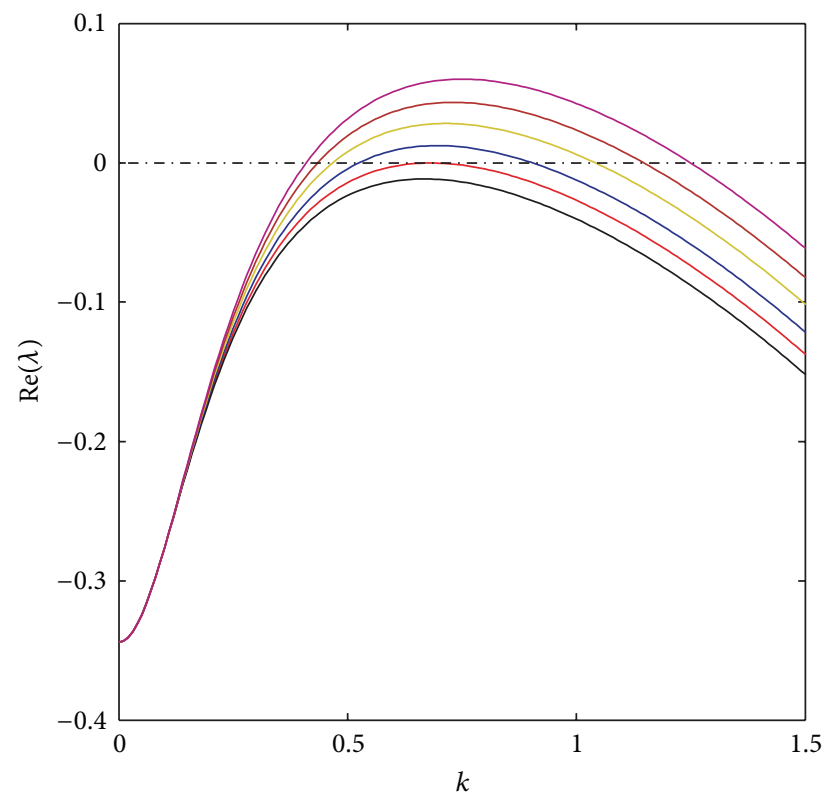

(c)

Figure 2: Dispersion relations for different parameters: in (a), curves of the real part of the eigenvalue are derived by taking $a_{21}=10$, $a_{12}=a_{22}=0.01$, and $a_{11}=0.05,0.1,0.15,0.18,0.201,0.25$ from top to bottom; in (b) $a_{21}=10, a_{11}=a_{22}=0.01$, and $a_{12}=$ $0.15,0.2,0.25,0.3,0.353,0.4$ from top to bottom; and in (c) $a_{21}=5.06, a_{11}=a_{12}=0.01$, and $a_{22}=0.35,0.4,0.45,0.508,0.558,0.608$ from top to bottom. Other parameters are fixed as in (21).

the homogeneous steady state $\mathbf{u}^{*}$ is expected to be unstable. These charts are obtained by fixed parameters in (21) as well as $a_{12}=a_{22}=0.01$ for Figure $1(\mathrm{a})$, $a_{11}=a_{22}=0.01$ for Figure 1(b), and $a_{11}=a_{12}=0.01$ for Figure 1(c).

From the mathematical viewpoint, the Turing bifurcation occurs when for the characteristic root of (13), $\operatorname{Im}(\lambda(\mu))=0$ and $\operatorname{Re}(\lambda(\mu))=0$ at $\mu=\mu_{c} \neq 0$. Next, we will look for the critical wave of spatial patterns and note the relationship of $\mu$ and the wave number $k$; that is, $\mu=k^{2}$ [26]. Thus, we only need to confirm that

$$
\min _{k} \operatorname{det}\left(\mathbf{G}_{\mathbf{u}}\left(\mathbf{u}^{*}\right)-k^{2} \Phi_{\mathbf{u}}\right)=0 .
$$

Then, the Turing bifurcation thresholds of parameters satisfy the following equation:

$$
\begin{aligned}
4 \operatorname{det} & \left(\mathbf{G}_{\mathbf{u}}\left(\mathbf{u}^{*}\right)\right) \operatorname{det}\left(\Phi_{\mathbf{u}}\left(\mathbf{u}^{*}\right)\right) \\
& =\left(\Phi_{11} \mathbf{G}_{22}+\Phi_{22} \mathbf{G}_{11}-\Phi_{21} \mathbf{G}_{12}-\Phi_{12} \mathbf{G}_{21}\right)^{2}
\end{aligned}
$$



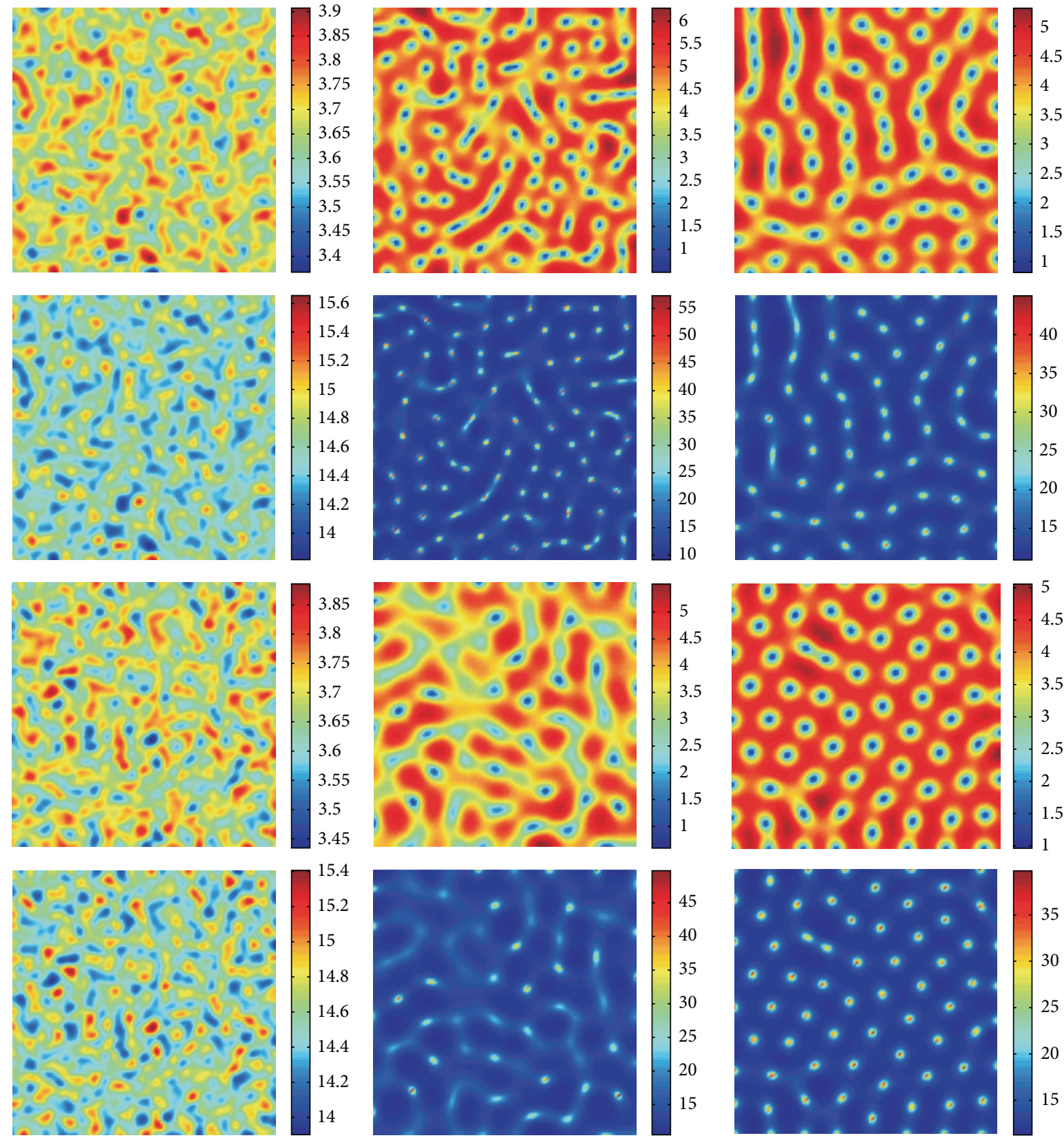

FIGURE 3: Snapshots of contour pictures of time evolution of $u_{1}$ and $u_{2}$. From top to bottom: patterns of $u_{1}, u_{2}$ for $a_{11}=0.05$ and of $u_{1}, u_{2}$ for $a_{11}=0.1$. From left to right: $2 \times 10^{3}$ iterations, $4 \times 10^{4}$ iterations, and $1 \times 10^{6}$ iterations. Other diffusion parameters: $a_{12}=a_{22}=0.01$ and $a_{21}=10$.

and the critical wavenumber $k_{c}$ satisfies

$$
k_{c}^{2}=\frac{\left(\Phi_{11} \mathbf{G}_{22}+\Phi_{22} \mathbf{G}_{11}-\Phi_{21} \mathbf{G}_{12}-\Phi_{12} \mathbf{G}_{21}\right)}{\left(2 \operatorname{det}\left(\Phi_{\mathbf{u}}\left(\mathbf{u}^{*}\right)\right)\right)}
$$

To well see the effect of the nonlinear diffusion, according to Turing parameter regions in Figure 1 we plot the dispersion relations in Figure 2. The critical parameter values in Figure 2(a) correspond to $a_{11}=0.201, a_{12}=0.353$ in Figure 2(b), and $a_{22}=0.558$ in Figure 2(c). In addition, we find that the lowest limit of wavenumber $k$ corresponding to the available
Turing modes $\operatorname{Re}(\lambda)>0$ turns small with $a_{11}$ increasing in Figure 2(a), with $a_{12}$ increasing in Figure 2(b), and with $a_{22}$ increasing in Figure 2(c).

\section{Pattern Formation}

In this section, using numerical methods, we perform numerical simulations of the system (1) in a two-dimensional space and illustrate that cross-diffusion and self-diffusion induce spatial patterns. Throughout this section, we assume that 

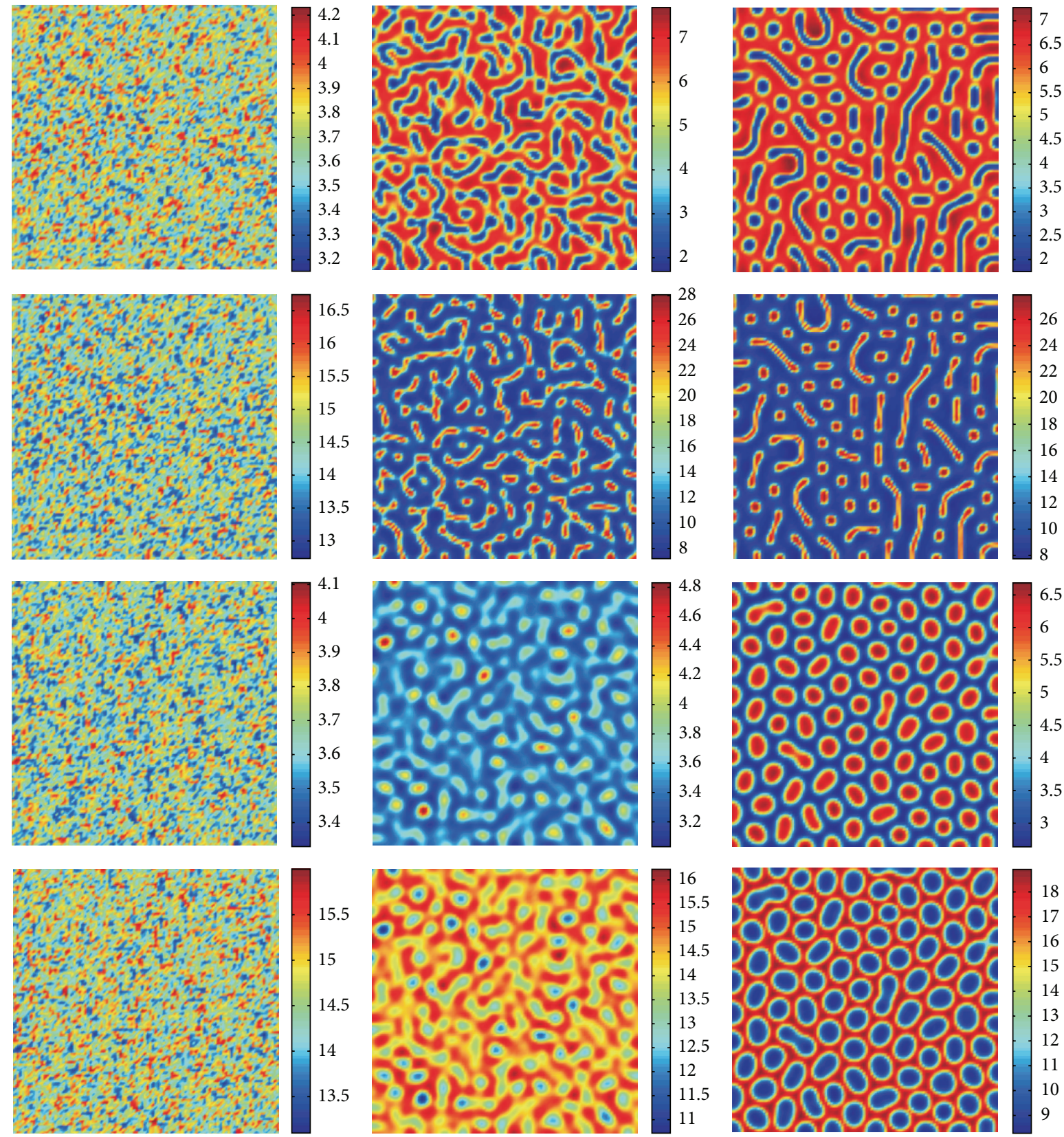

FIGURE 4: Snapshots of contour pictures of time evolution of $u_{1}$ and $u_{2}$. From top to bottom: patterns of $u_{1}, u_{2}$ for $a_{12}=0.15$ and of $u_{1}, u_{2}$ for $a_{12}=0.3$. From left to right: $2 \times 10^{3}$ iterations, $4 \times 10^{4}$ iterations, and $1 \times 10^{6}$ iterations. Other diffusion parameters: $a_{11}=a_{22}=0.01$ and $a_{21}=10$.

the region of the system $(1)$ is $[0, L] \times[0, L] \subset \mathbb{R}^{2}$. Hence, according to the definition of the eigenvalue $\mu_{i}$ in Notation 2 , we can obtain $\mu_{i}=((i-1) \pi / L)^{2}$. To numerically solve partial differential equations, we first have to discretize the spacetime of the system (1). The region of $[0, L] \times[0, L]$ is solved in a discrete domain with $M \times N$ lattice sites. The length of the lattices is defined by a constant $h$. The time is also discrete by a constant step $\tau$. All our numerical simulations employ the Neumann boundary condition. Here, we use the standard five-point approximation for the two-dimensional Laplacian derivative and the time evolution is solved by using the Euler method. More precisely, the value $\left(u_{1, i, j}^{n+1}, u_{2, i, j}^{n+1}\right)$ at the time $(n+1) \tau$ at the mesh position $\left(x_{i}, y_{i}\right)$ is obtained by

$$
\begin{aligned}
& u_{1, i, j}^{n+1}=u_{1, i, j}^{n}+\tau \Delta \Phi_{1}+\tau G_{1}\left(u_{1, i, j}^{n}, u_{2, i, j}^{n}\right), \\
& u_{2, i, j}^{n+1}=u_{2, i, j}^{n}+\tau \Delta \Phi_{2}+\tau G_{2}\left(u_{1, i, j}^{n}, u_{2, i, j}^{n}\right),
\end{aligned}
$$

with Laplacian defined by

$$
\Delta \Phi_{1}=\frac{\Phi_{1}\left(u_{1, i+1, j}, u_{2, i+1, j}\right)+\Phi_{1}\left(u_{1, i-1, j}, u_{2, i-1, j}\right)}{h^{2}}
$$



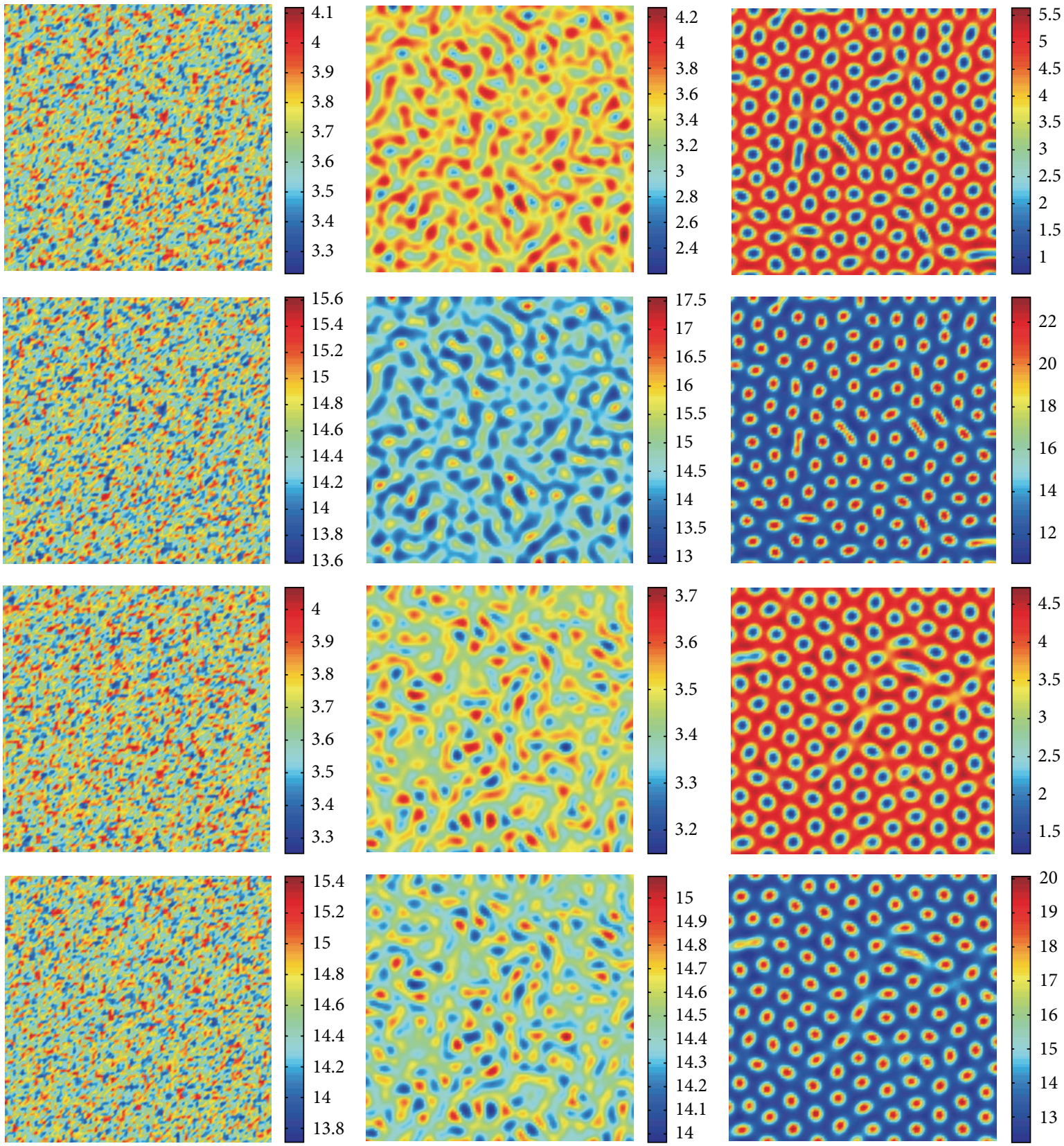

FIGURE 5: Snapshots of contour pictures of time evolution of $u_{1}$ and $u_{2}$. From top to bottom: patterns of $u_{1}, u_{2}$ for $a_{22}=0.35$ and of $u_{1}, u_{2}$ for $a_{22}=0.508$. From left to right: $2 \times 10^{3}$ iterations, $4 \times 10^{4}$ iterations, and $1 \times 10^{6}$ iterations. Other diffusion parameters: $a_{11}=a_{12}=0.01$ and $a_{21}=5.06$.

$$
\begin{aligned}
& +\frac{\Phi_{1}\left(u_{1, i, j+1}, u_{2, i, j+1}\right)+\Phi_{1}\left(u_{1, i, j-1}, u_{2, i, j-1}\right)}{h^{2}} \\
& -\frac{4 \Phi_{1}\left(u_{1, i, j}, u_{2, i, j}\right)}{h^{2}} .
\end{aligned}
$$

In this paper, we set $h=1, \tau=0.001$, and $M=N=100$. The initial data of the system (1) is taken as a uniformly distributed random perturbation in order of $1 \times 10^{-4}$ around the homogeneous equilibrium state $\mathbf{u}^{*}$. More precisely,

$$
u_{1}(x, 0)=u_{1}^{*}+\eta_{1}(x), \quad u_{2}(x, 0)=u_{2}^{*}+\eta_{2}(x),
$$

where $\eta_{1}, \eta_{2} \in\left[-1 \times 10^{-4}, 1 \times 10^{-4}\right]$. We simulate different patterns according to the dispersion relations in Figure 2.

In Figure 3, we show the evolution of the spatial patterns of the prey and the predators at $2 \times 10^{3}, 4 \times 10^{4}$, and $1 \times 10^{6}$ 
iterations for $a_{11}=0.05,0.1$ when we set $a_{21}=10, a_{12}=0.01$, and $a_{22}=0.01$. One can see that the patterns arise from random initial conditions. After the cold spot patterns for the prey and the hot spot patterns for the predator arise, they turn steadily with time until these patterns are temporally independent. In addition, for $a_{11}=0.1$ the cold spot patterns for the prey and the hot spot patterns for the predator are looser compared with those for $a_{11}=0.05$.

In Figure 4, we fix $a_{11}=0.01, a_{22}=0.01$, and $a_{21}=$ 10 and obtain the spatial patterns of species $u_{1}, u_{2}$ of time evolution for $a_{12}=0.15$ and $a_{12}=0.3$. For the case of $a_{12}=0.15$, the random initial distribution leads to the formation of irregular patterns. After a long time evolution, we find that the cold spot-strip patterns emerge for the prey $u_{1}$ and that the hot spot-strip patterns for the predator $u_{2}$ arise. However, in the case of $a_{12}=0.3$, the steady patterns of the prey $u_{1}$ consist of hot spots in a bigger size, while the steady patterns of the predator $u_{2}$ are in the formation of bigger cold spots.

In Figure 5, diffusion parameters are set as $a_{11}=a_{12}=0$ and $a_{21}=5.06$. We plot the patterns for $a_{22}=0.35$ and $a_{22}=$ 0.508 , respectively. For both cases, one can see that as time goes on, the cold spot patterns of the prey $u_{1}$ and the hot spot patterns of the predator $u_{2}$ ultimately form.

\section{Conclusion and Discussion}

In this paper, we have studied the prey-predator model with the nonlinear diffusions including normal diffusion, crossdiffusion, and self-diffusion. By applying the mathematical analysis and suitable numerical simulations, we obtain the sufficient conditions of the formation of Turing patterns for this nonlinear diffusion and illustrate Turing parameter regions and Turing patterns when some parameters in system (1) are set.

In our results, we have provided Theorems 1 and 3 to demonstrate that for the nonlinear diffusion including self-diffusion and the cross-diffusion of the predator, the parameter $a_{21}$ plays an important role to induce Turing instability. Furthermore, if $a_{21}=0$, then the homogeneous equilibrium state $\mathbf{u}^{*}$ is always stable; that is, the system (1) has no Turing patterns. By performing numerical simulations, we find the Turing parameter regions of the interaction between cross-diffusion $a_{21}$ and other diffusion terms including cross-diffusion of the other species and self-diffusions. Besides, according to these parameter regions, we show the corresponding dispersion relations and the corresponding patterns. These results indicate that Turing patterns can emerge through the interaction between the cross-diffusion $a_{21}$ and self-diffusion as well as other cross-diffusions in the system (1).

It is well known that for a prey-predator system, the formation of patterns can occur by introducing the crossdiffusion. However, our results further show that under condition (see Theorem 3), self-diffusion can produce Turing patterns.

\section{Acknowledgments}

The authors would like to thank the referees for their careful reading and constructive comments to improve their paper and writing. This work is supported by Doctoral Initial Foundation of Nanchang University, China (no. 06301004), and the Youth Natural Science Foundation of China (no. 61304161).

\section{References}

[1] V. Volterra, "Variazioni e fluttuazioni del numero d'individui in specie conviventi," Memorie Accademia dei Lincei, vol. 2, pp. 31$113,1926$.

[2] J. D. Murray, Mathematical Biology: Spatial Models and Biomedical Applications, Springer, New York, NY, USA, 2003.

[3] E. A. McGehee and E. Peacock-López, "Turing patterns in a modified Lotka-Volterra model," Physics Letters A, vol. 342, no. 1-2, pp. 90-98, 2005.

[4] J. D. Murray, Mathematical Biology I: An introduction, vol. 17, Springer, New York, NY, USA, 3rd edition, 2002.

[5] E. H. Colombo and C. Anteneodo, "Nonlinear diffusion effects on biological population spatial patterns," Physical Review E, vol. 86, Article ID 036215, 2012.

[6] W. Wang, Q.-X. Liu, and Z. Jin, "Spatiotemporal complexity of a ratio-dependent predator-prey system," Physical Review E, vol. 75, no. 5, Article ID 051913, 9 pages, 2007.

[7] M. A. Fuentes, M. N. Kuperman, and V. M. Kenkre, "Nonlocal interaction effects on pattern formation in population dynamics," Physical Review Letters, vol. 91, Article ID 158104, 2003.

[8] L. Xue, "Pattern formation in a predator-prey model with spatial effect," Physica A, vol. 391, pp. 5987-5996, 2012.

[9] J. A. R. Da Cunha, A. A. Penna, F. A. Oliveira, and Pattern formation a, "nd coexistence domains for a nonlocal population dynamics," Physical Review E, vol. 83, Article ID 015201, 2011.

[10] D. Alonso, F. Bartumeus, and J. Catalan, "Mutual interference between predators can give rise to Turing spatial patterns," Ecology, vol. 83, pp. 28-34, 2002.

[11] W. Wang, Y. Lin, F. Rao, L. Zhang, and Y. Tan, "Pattern selection in a ratio-dependent predator-prey model," Journal of Statistical Mechanics, vol. 2010, Article ID P11036, 2010.

[12] A. M. de Roos, E. McCauley, W. G. Wilson, and Pattern formation a, "nd the spatial scale of interaction between predators and their prey," Theoretical Population Biology, vol. 53, pp. 108-130, 1998.

[13] M. Baurmann, T. Gross, and U. Feudel, "Instabilities in spatially extended predator-prey systems: spatio-temporal patterns in the neighborhood of Turing-Hopf bifurcations," Journal of Theoretical Biology, vol. 245, no. 2, pp. 220-229, 2007.

[14] V. K. Vanag, I. R. Epstein, and Others, "Out-of-phase oscillatory Turing patterns in a bistable reaction-diffusion system," Physical Review E, vol. 71, Article ID 66212, 2005.

[15] B. I. Henry, T. A. M. Langlands, and S. L. Wearne, "Turing pattern formation in fractional activator-inhibitor systems," Physical Review E, vol. 72, no. 2, Article ID 026101, 14 pages, 2005.

[16] A. M. Turing, “The chemical basis of mokphogenesis," Philosophical Transactions of the Royal Society of London B, vol. 237, pp. 37-72, 1952. 
[17] Y. Lou, W.-M. Ni, and Y. Wu, "On the global existence of a crossdiffusion system," Discrete and Continuous Dynamical Systems, vol. 4, no. 2, pp. 193-203, 1998.

[18] Y. Li and C. Zhao, "Global existence of solutions to a crossdiffusion system in higher dimensional domains," Discrete and Continuous Dynamical Systems A, vol. 12, no. 2, pp. 185-192, 2005.

[19] Y. S. Choi, R. Lui, and Y. Yamada, "Existence of global solutions for the Shigesada-Kawasaki-Teramoto model with strongly coupled cross-diffusion," Discrete and Continuous Dynamical Systems A, vol. 10, no. 3, pp. 719-730, 2004.

[20] S.-A. Shim, "Uniform boundedness and convergence of solutions to the systems with cross-diffusions dominated by selfdiffusions," Nonlinear Analysis: Real World Applications, vol. 4, no. 1, pp. 65-86, 2003.

[21] Y. Cai and W. Wang, "Spatiotemporal dynamics of a reactiondiffusion epidemic model with nonlinear incidence rate," Journal of Statistical Mechanics, vol. 2011, Article ID P02025, 2011.

[22] C. Tian, Z. Ling, and Z. Lin, "Turing pattern formation in a predator-prey-mutualist system," Nonlinear Analysis: Real World Applications, vol. 12, no. 6, pp. 3224-3237, 2011.

[23] W. Wang, Y. Cai, M. Wu, K. Wang, and Z. Li, "Complex dynamics of a reaction-diffusion epidemic model," Nonlinear Analysis: Real World Applications, vol. 13, no. 5, pp. 2240-2258, 2012.

[24] R. Ruiz-Baier and C. Tian, "Mathematical analysis and numerical simulation of pattern formation under cross-diffusion," Nonlinear Analysis: Real World Applications, vol. 14, no. 1, pp. 601-612, 2013.

[25] X. Guan, W. Wang, and Y. Cai, "Spatiotemporal dynamics of a Leslie-Gower predator-prey model incorporating a prey refuge," Nonlinear Analysis: Real World Applications, vol. 12, no. 4, pp. 2385-2395, 2011.

[26] C. Tian, Z. Lin, and M. Pedersen, "Instability induced by crossdiffusion in reaction-diffusion systems," Nonlinear Analysis: Real World Applications, vol. 11, no. 2, pp. 1036-1045, 2010.

[27] G. Galiano and J. Velasco, "Competing through altering the environment: a cross-diffusion population model coupled to transport-Darcy flow equations," Nonlinear Analysis: Real World Applications, vol. 12, no. 5, pp. 2826-2838, 2011.

[28] J.-F. Zhang, W.-T. Li, and Y.-X. Wang, "Turing patterns of a strongly coupled predator-prey system with diffusion effects," Nonlinear Analysis: Theory, Methods \& Applications A, vol. 74, no. 3, pp. 847-858, 2011.

[29] A. Okubo and S. A. Levin, Diffusion and Ecological Problems: Modern Perspectives, vol. 14, Springer, New York, NY, USA, 2002.

[30] S.-A. Shim, "Long-time properties of prey-predator system with cross-diffusion," Communications of the Korean Mathematical Society, vol. 21, no. 2, pp. 293-320, 2006.

[31] S. Xu, "Existence of global solutions for a predator-prey model with cross-diffusion," Electronic Journal of Differential Equations, vol. 2008, pp. 1-14, 2008. 


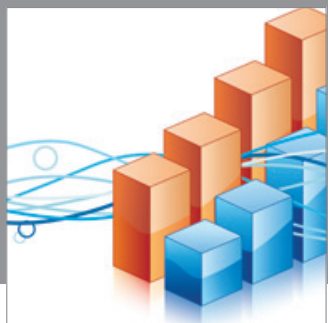

Advances in

Operations Research

mansans

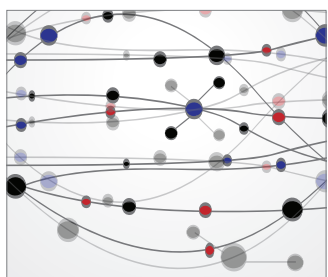

The Scientific World Journal
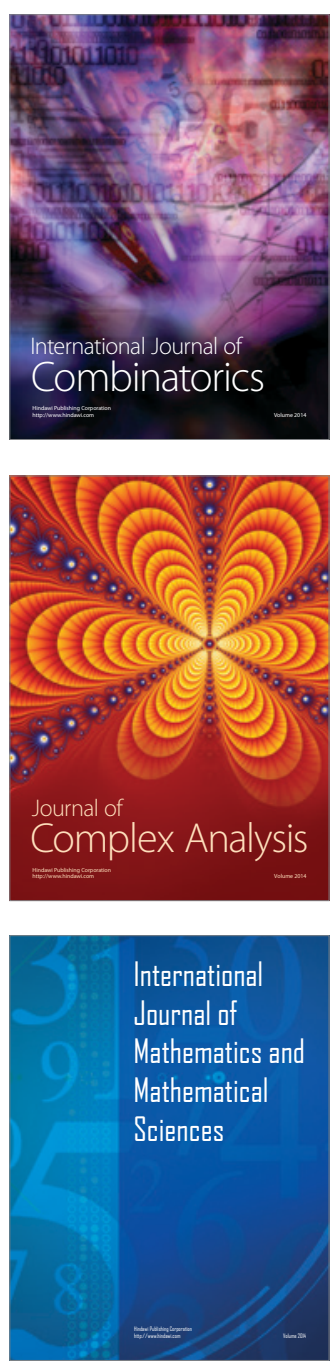
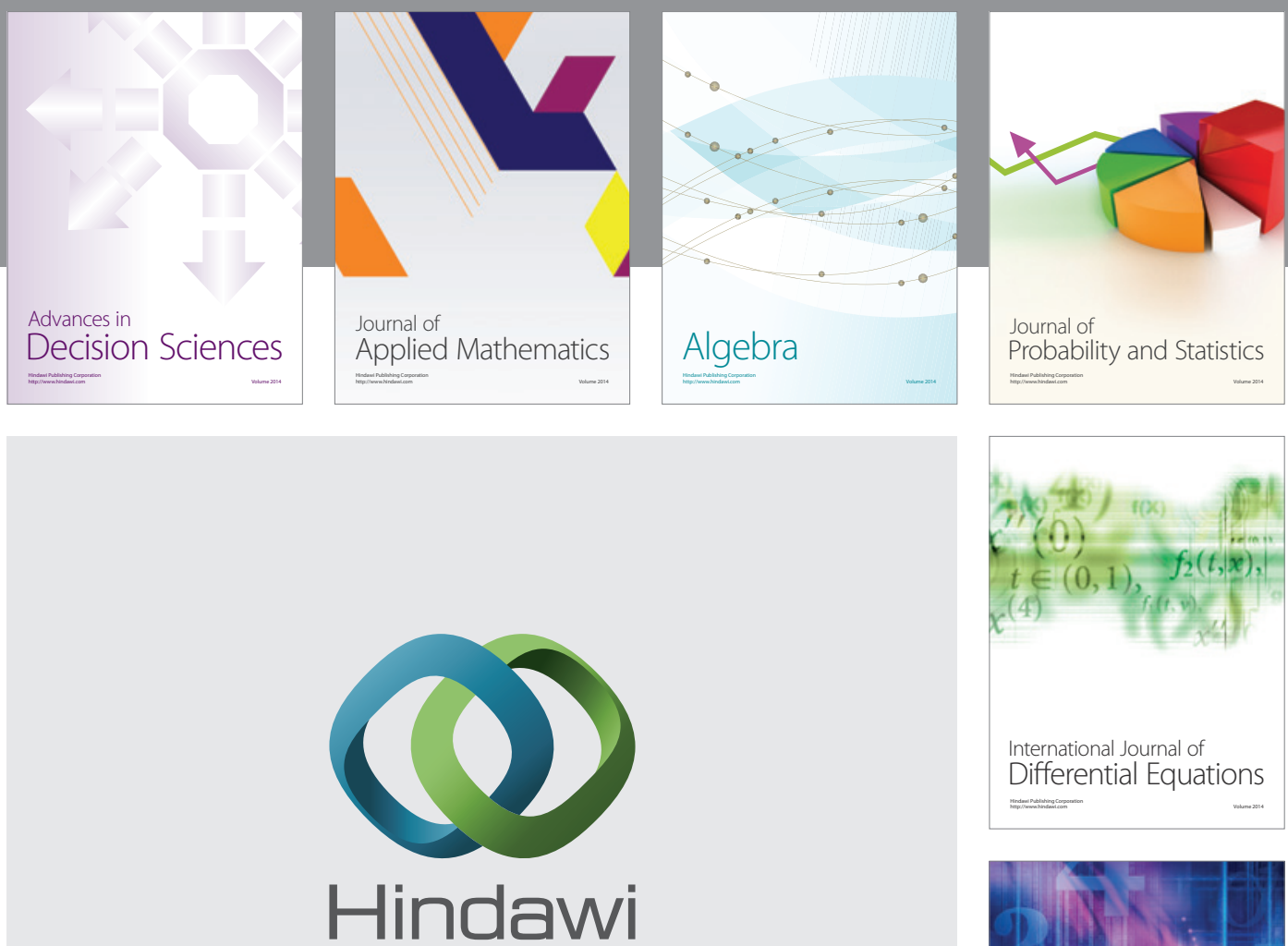

Submit your manuscripts at http://www.hindawi.com
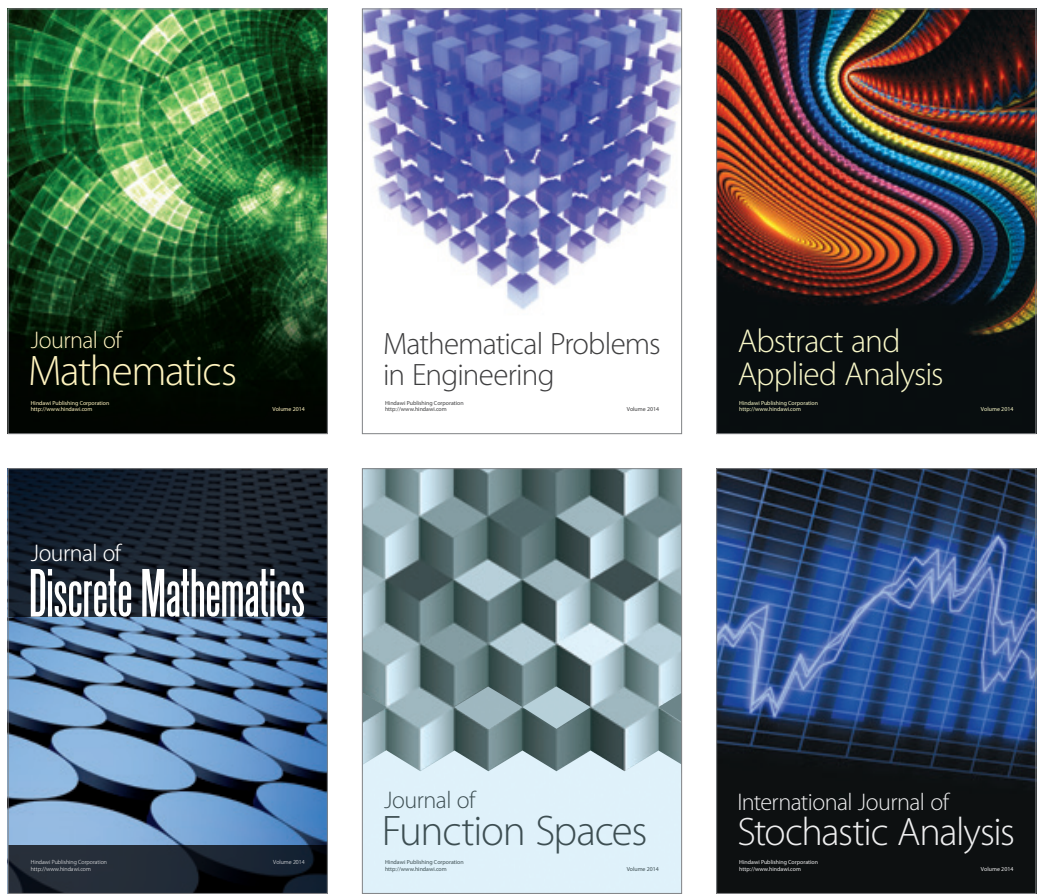

Journal of

Function Spaces

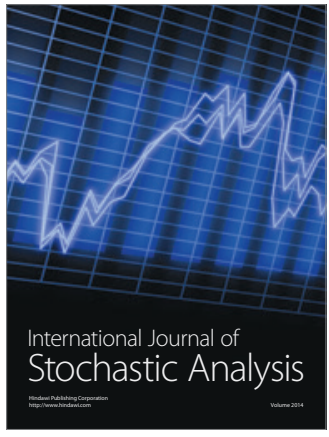

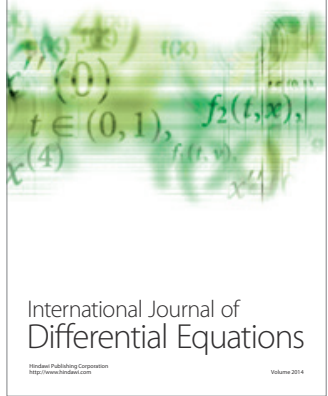
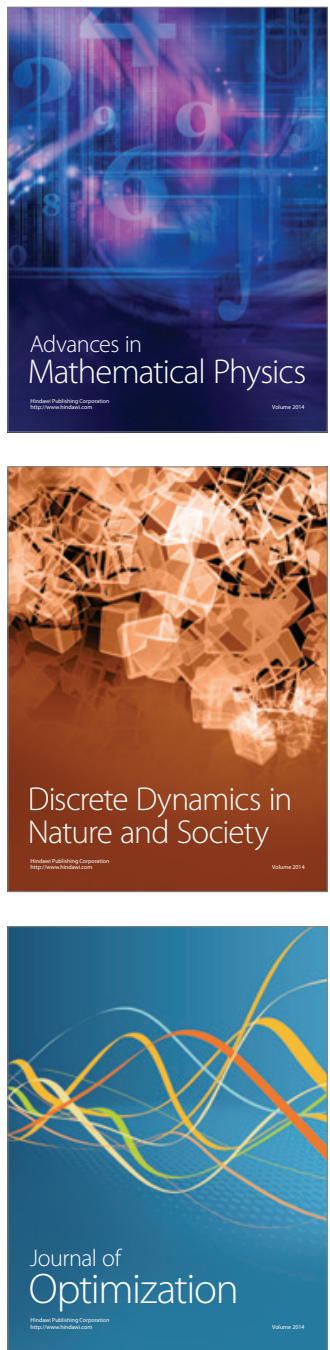